\title{
Prioritization of Patients for Comprehensive Medication Review By a Clinical Pharmacist in Family Medicine
}

\author{
Joseph P. Vande Griend, PharmD, Joseph J. Saseen, PharmD, Debra Bislip, MD, \\ Caroline Emsermann, PhD, Colleen Conry, MD, and Wilson D. Pace, MD
}

Background: This pilot study describes and evaluates the clinical pharmacy priority (CP2) score. We hypothesize that patients with high CP2 scores are more likely to receive a medication recommendation after comprehensive medication review (CMR) than patients with lower scores. Prioritization of patients for CMR by a clinical pharmacist in family medicine could enhance the provision of interprofessional care within the patient-centered medical home.

Methods: The CP2 score was developed collaboratively by the research team and is derived from 11 patient-specific factors extracted from the electronic health record. To evaluate the utility of the score, CMR was performed prospectively by a clinical pharmacist for patients with appointments between 0ctober 1 and December 31, 2012, at 2 University of Colorado family medicine clinics.

Results: CMR was performed for 1107 patient appointments. Of these, 101 were identified as having received a medication recommendation from the clinical pharmacist. For patients with a CP2 score of 0 to 2,2 of 588 charts $(0.3 \%)$ reviewed received a recommendation (level 1). The proportion increased to 37 of $358(10.3 \%)$ for scores of 3 to 7 (level 2), 40 of 119 (33.6\%) for scores of 8 to 10 (level 3), and 22 of $42(52.4 \%)$ for scores of $\geq 11$ (level 4). Compared with CP2 scores in level 1, patient appointments were more likely to receive a medication recommendation after CMR in level 2 (relative risk [RR], 30.4; 95\% confidence interval [CI], 7.4-125.3), in level 3 (RR, 98.8; 95\% CI, 24.2-403.3), and in level 4 (RR, 154; 95\% CI, 37.5-632.8).

Conclusions: Patients with higher CP2 scores were more likely to receive a medication recommendation after CMR by a clinical pharmacist than patients with lower scores. The CP2 score could be used by clinical pharmacists in family medicine to enhance the efficient and effective delivery of interprofessional care. (J Am Board Fam Med 2015;28:418-424.)

Keywords: Delivery of Health Care, Family Practice, Health Care Rationing, Medication Therapy Management

An offsite clinical pharmacy service has been provided at a small, off-campus University of Colorado family medicine clinic (the Park Meadows Clinic) since July 2011. ${ }^{1}$ A clinical pharmacist uses the electronic health record (EHR) to manually review patients with an appointment scheduled during the upcoming week. When a drug therapy problem is identified, the clinical pharmacist writes a clinical

This article was externally peer reviewed.

Submitted 3 November 2014; revised 21 January 2015; accepted 26 January 2015.

From the Department of Clinical Pharmacy, University of Colorado Skaggs School of Pharmacy and Pharmaceutical Sciences, Aurora (JPVG, JJS), and the Department of Family Medicine, University of Colorado School of Medicine, Aurora (JPVG, JJS, DB, CE, CC, WDP). pharmacy consultation note in the medical record before the patient visit. The note becomes a permanent part of the medical record and is sent to the provider, who can then act on identified problems as appropriate during the patient visit. This offsite clinical pharmacy model has enhanced interprofes-

Funding: This study was funded by the University of Colorado Hospital/University of Colorado School of Medicine Quality Improvement and Small Grants Program.

Conflict of interest: none declared.

Corresponding author: Joseph P. Vande Griend, PharmD, BCPS, CGP, Department of Clinical Pharmacy and Family Medicine, University of Colorado, Anschutz Medical Campus, Skaggs School of Pharmacy and Pharmaceutical Sciences, Mail Stop C238, 12850 E. Montview Blvd, Room V20-2127, Aurora, CO 80045 (E-mail: joseph.vandegriend@ ucdenver.edu). 
sional delivery of care, resulting in $>300$ medication recommendations, of which $69.8 \%$ were implemented, and $>\$ 50,000$ of direct cost savings after the first year. ${ }^{1}$ The clinical pharmacist provided the service for 118 hours the first year, for an estimated pharmacist cost of $\$ 8500$, resulting in a return on investment of $>\$ 40,000 .{ }^{1}$ Despite very positive financial and health care quality benefits, there was a desire to expand the service without incurring significant additional cost from pharmacist time. The process of identifying patients with drug therapy problems required individual auditing and evaluation of all patient appointments; it could take up to 6 hours to review a clinic schedule of 100 patient appointments per week. Similar to most family medicine clinics, patients at the Park Meadows Clinic include healthy newborns and young and middle-aged patients with no medication therapy, young and middle-aged adults with multiple comorbidities and complex medication regimens, and geriatric patients with varying levels of health and varying medication regimens. ${ }^{2,3}$ Many of these patients do not have drug therapy problems. A process to more efficiently prioritize patients for comprehensive medication review (CMR) was needed.

After a thorough review of the primary literature, no prioritization tool or strategy was identified. Two tools that gather information from individual patients have been developed. ${ }^{4,5}$ Both require patient input and gather information using a series of medication use questions, including number of medications, adherence, and cost. Both seem able to identify patients with a higher likelihood of having $\geq 1$ medication-related problem, but both are labor intensive and would not be able to be incorporated into an EHR. A basic "complexity score," calculated by adding the number of chronic conditions and the number of medications a patient takes, for elderly primary care patients has also been described. ${ }^{6}$ This tool has not been used to assess the need for medication review by a clinical pharmacist. The very basic nature of this score may make differentiating the need for CMR within a group of more complex patients difficult. A software-based tool used in the inpatient setting has been published. ${ }^{7}$ It pulls clinical information and medication use data from the EHR to identify patients as low, medium, or high risk of having an adverse drug event. The tool uses numerous prespecified clinical flags for high-risk medication use and other adverse drug event risk factors. It seems to be useful for its intended purpose but was developed specifically to target adverse drug events in the inpatient setting, and many of the clinical flags are not relevant to the ambulatory environment.

Therefore the primary objective of this study was to develop a tool, called the Clinical Pharmacy Priority (CP2) score, that would identify patients in a family medicine clinic who are more likely to have drug therapy problems and prioritize them for CMR. The score would be calculated from patientspecific data in the EHR. The presence of chronic disease, uncontrolled chronic disease, higher number of medications, and age would result in a higher score. We hypothesized that patients with higher scores would be more likely to receive a medication recommendation after CMR than patients with lower scores. We also hypothesized that this tool would save clinical pharmacist time by increasing efficiency.

\section{Methods}

This study was approved by the Colorado Multiple Institutional Review Board. The CP2 score tool was developed a priori using patient-specific factors available in the EHR. The following criteria were used when deciding which patient-specific factors to include in the tool: (1) easily identified and pulled from the EHR for reporting; (2) believed to be associated with a higher likelihood of drug therapy problems based on clinical judgment (eg, uncontrolled diabetes or uncontrolled hypertension); and (3) defined a patient population that is subject to health care quality indicator evaluation for which a clinical pharmacist could have a positive impact (eg, diabetes, age $>65$ years). The initial list of patient-specific factors was developed using these criterion by Dr. Vande Griend and Dr. Pace and included 10 patient-specific factors. This list then was circulated and discussed individually with the other authors. Ultimately, the presence of a depression diagnosis was added, resulting in a total of 11 patient-specific factors. We determined point allotment based on our clinical knowledge and experience. The score ranged from 0 to a maximum of 21 points (Table 1).

To calculate CP2 scores, information technology professionals developed code to extract applicable patient-specific data and International Classification of Diseases, 9th Revision (ICD-9), codes 
Table 1. The Clinical Pharmacy Priority (CP2) Score Algorithm

\begin{tabular}{|c|c|}
\hline Criteria & Score \\
\hline \multicolumn{2}{|l|}{ Age (years) } \\
\hline$<65$ & 1 \\
\hline $65-75$ & 2 \\
\hline$>75$ & 3 \\
\hline \multicolumn{2}{|l|}{ Diagnosis (code) } \\
\hline Diabetes mellitus (250.X) & 2 \\
\hline $\begin{array}{l}\text { Chronic obstructive pulmonary disease } \\
\quad(491 . X, 492 . X, 496 . X)\end{array}$ & 1 \\
\hline $\begin{array}{l}\text { Hypertension (401.X to } 405 . \mathrm{X} \\
\text { inclusive) }\end{array}$ & 2 \\
\hline $\begin{array}{l}\text { Cardiovascular disease }(410 . \mathrm{X}-414 . \mathrm{X} \\
\text { inclusive, } 429.2,434.0,434.00 \\
\text { 434.01,435.8, 435.9, 436, 437.0, } \\
\text { 437, 434.9x, 435, 437.1, 440.0, } \\
\text { 441.3, 443, 440.8, 440.9, 441.4, } \\
\text { 443.89, 443.9) }\end{array}$ & 2 \\
\hline Heart failure (428.X) & 1 \\
\hline Depression (311.x, 296.2x, 296.3x) & 1 \\
\hline $\begin{array}{l}\text { Estimated glomerular filtration rate }<45 \\
\mathrm{~mL} / \mathrm{min}\end{array}$ & 1 \\
\hline \multicolumn{2}{|l|}{ Hemoglobin $\mathrm{A}_{1 \mathrm{c}}$} \\
\hline$>7.9 \%$ & 1 \\
\hline$>8.9 \%$ & 2 \\
\hline \multicolumn{2}{|l|}{$\mathrm{BP}$} \\
\hline $\begin{array}{l}\text { Last } \mathrm{BP}>140 / 90 \mathrm{mmHg} \text { and last } 3 \\
\text { averaged }>140 / 90 \mathrm{mmHg}\end{array}$ & 1 \\
\hline $\begin{array}{l}\text { Last } \mathrm{BP}>160 / 100 \mathrm{mmHg} \text { and last } 3 \\
\text { average }>160 / 100 \mathrm{mmHg}\end{array}$ & 2 \\
\hline \multicolumn{2}{|l|}{ Active items on medication list } \\
\hline$<3$ & 0 \\
\hline $3-5$ & 1 \\
\hline $6-9$ & 2 \\
\hline$>9$ & 4 \\
\hline
\end{tabular}

BP, blood pressure.

from the EPIC EHR (Epic Systems Corp., Verona, WI) and assign points. The list of all patient appointments and the corresponding CP2 scores (including the individual point assignment) were extracted from the EHR in an automated fashion as a cross-section each Friday for the upcoming week. To determine whether a higher CP2 score was more likely to result in the identification of a drug therapy problem, a clinical pharmacist (JPVG) conducted CMR weekly at the University of Colorado Park Meadows Clinic and the University of Colorado Boulder family medicine clinics from October 2012 through December 2012 using the list of all patient appointments with a corresponding CP2 score and individual point assignment. At that time, these 2 clinics combined had 6 physician providers and 4 nonphysician providers, for a total of 8.8 full-time employees. In total, CMR was performed on 3 of every 4 clinic days, resulting in a $75 \%$ sample.

\section{Statistical Analysis}

For the analyses, all patient appointments reviewed by the clinical pharmacist between October 2012 and December 2012 were used. Patient appointments reviewed during this time frame were identified as having received a medication recommendation if a clinical pharmacist note was written during the October 2012 and December 2012 time frame or if a clinical pharmacist note was written previously when clinical pharmacy services were first established at the Park Meadows Clinic in July 2011. The individual components of the CP2 scoring criteria and the frequency of medication recommendation for each component were summarized. The $\chi^{2}$ test of association was performed to evaluate the difference in frequency of medication recommendation with the CP2 scoring criterion. The number of patient appointments receiving a medication recommendation also was summarized for each of the CP2 scores. These data then were organized into 4 levels by looking at the CP2 scores and creating logical cutoff points according to similar medication recommendation rates. Relative risk (RR) was calculated for levels 2, 3, and 4, with level 1 serving as the referent. In addition, a logistic regression analysis of the outcomes "received recommendation" or "did not receive recommendation" was performed, adjusting for CP2 score as an ordinal measure, to determine whether increased $\mathrm{CP} 2$ score is linearly associated with receiving a recommendation.

In addition, patient appointments with low CP2 scores were analyzed in an attempt to identify patient characteristics, separately and in combination, that could be associated with receipt of a recommendation. Patient characteristics were identified in an exploratory manner and were selected if a statistical association between these combined characteristics and medication recommendation received was determined. For these analyses, the $\chi^{2}$ test of association was used. All analyses were run using SAS software version 9.3 (SAS Institute, Inc., Cary, NC). Statistical significance was determined at $\alpha=0.05$. 
Table 2. Clinical Pharmacy Priority (CP2) Scoring Criteria Among Those Receiving and Not Receiving a Medication Recommendation

\begin{tabular}{|c|c|c|c|}
\hline & Recommendation $(\mathrm{n}=101)$ & No Recommendation $(\mathrm{n}=1006)$ & $P$ Value \\
\hline Age (years) & & & $<.0001$ \\
\hline$<65$ & $40.6 \%$ & $76.8 \%$ & \\
\hline $65-75$ & $38.6 \%$ & $17.4 \%$ & \\
\hline$>75$ & $20.8 \%$ & $5.8 \%$ & \\
\hline \multicolumn{4}{|l|}{ Diagnosis } \\
\hline COPD & $7.9 \%$ & $3.6 \%$ & .0332 \\
\hline Diabetes & $48.5 \%$ & $6.4 \%$ & $<.0001$ \\
\hline Hypertension & $84.2 \%$ & $27.1 \%$ & $<.0001$ \\
\hline Vascular disease & $26.7 \%$ & $5.4 \%$ & $<.0001$ \\
\hline Heart failure & $5 \%$ & $1 \%$ & .001 \\
\hline Depression & $22.8 \%$ & $14.7 \%$ & .0326 \\
\hline $\mathrm{eGFR}<45 \mathrm{~mL} / \mathrm{min}$ & $9.9 \%$ & $2.5 \%$ & $<.0001$ \\
\hline Hemoglobin $\mathrm{A}_{1 \mathrm{c}}>7.9 \%$ & $11.9 \%$ & $0.9 \%$ & $<.0001$ \\
\hline Stage 1 or stage 2 hypertension* & $19.8 \%$ & $5.6 \%$ & $<.0001$ \\
\hline Items on medication list & & & $<.0001$ \\
\hline$<3$ & $2 \%$ & $34.2 \%$ & \\
\hline $3-5$ & $9.9 \%$ & $25.7 \%$ & \\
\hline $6-9$ & $25.7 \%$ & $21 \%$ & \\
\hline$\geq 9$ & $61.4 \%$ & $19.1 \%$ & \\
\hline
\end{tabular}

*Stage 1 hypertension $=$ blood pressure 140-159/90-99 mmHg; stage 2 hypertension $=$ blood pressure $\geq 160 / 100 \mathrm{mmHg}$. COPD, chronic obstructive pulmonary disease; eGFR, estimated glomerular filtration rate.

\section{Results}

During the October to December 2012 time frame, CMR was performed by the clinical pharmacist for 1107 patient appointments. The mean \pm standard deviation age of the entire population was $51 \pm$ 19.4 years. In total, 101 patient appointments were identified as having received a medication recommendation from a clinical pharmacist: 54 patient appointments during the October to December 2012 time frame and 47 patient appointments with a medication recommendation since July 2011. Patient characteristics based on the $\mathrm{CP} 2$ scoring criteria for all patients receiving a CMR are described in Table 2. The distribution of patient appointments and medication recommendation rates within each of the 4 levels of CP2 scores are shown in Table 3.

For patient appointments with a CP2 score of 0 to 2 (level 1$), 2$ of 588 charts $(0.3 \%)$ reviewed received a medication recommendation. The proportion that received a medication recommendation increased to 37 of 358 , or $10.3 \%$, for scores of 3 to 7 (level 2); to 40 of 119 , or $33.6 \%$, for scores of 8 to 10 (level 3); and 22 of 42 , or $52.4 \%$, for scores of $\geq 11$ (level 4). Compared with CP2 scores in level 1 , patient appointments were more likely to receive a medication recommendation after CMR in level 2 (RR, 30.4; 95\% confidence interval [CI], 7.4-125.3), in level 3 (RR, 98.8; 95\% CI, 24.2403.3), and in level 4 (RR, 154; 95\% CI, 37.5632.8). Using logistic regression, the odds of a patient appointment receiving a medication recommendation after CMR increased with increasing CP2 score $(P<.0001)$. Drug therapy problems identified and the most common medication recommendations made during these patient appointments are shown in Table 4. Overall, 223 medication recommendations were made by the clinical pharmacist in response to the 101 patient appointments, and 132 (59.2\%) were accepted and implemented.

Among patients with a level 2 CP2 score (score of 3 to 7), those who received a recommendation were more likely to have a diagnosis of diabetes mellitus $(21.6 \%$ vs $8.1 \% ; P=.0149)$, a diagnosis of hypertension $(78.4 \%$ vs $56.4 \% ; P=.0101)$, and a higher average $\mathrm{CP} 2$ score (5.35 vs 4.78; $P=.0169$ ). Exploratory analysis identified that patients with a level 2 CP2 score, a diagnosis of hypertension or diabetes mellitus, and either $\geq 6$ medications on their list or blood pressure $>140 / 90 \mathrm{mmHg}$ were more likely to receive a medication intervention 
Table 3. Clinical Pharmacy Priority (CP2) Score Levels and Medication Recommendation Rate

\begin{tabular}{ccc}
\hline CP2 Score & $\begin{array}{c}\text { Patient } \\
\text { Appointments } \\
\text { (n) }\end{array}$ & $\begin{array}{c}\text { Medication } \\
\text { Recommendation } \\
\text { Rate (\%) }\end{array}$ \\
\hline Level 1 & & \\
0 & 290 & 0 \\
1 & 178 & 0.6 \\
2 & 120 & 0.8 \\
Level 2 & & \\
3 & 73 & 4.1 \\
4 & 91 & 8.8 \\
5 & 75 & 9.3 \\
6 & 58 & 19 \\
7 & 61 & 13.1 \\
Level 3 & & \\
8 & 56 & 28.6 \\
9 & 45 & 37.8 \\
10 & 18 & 38.9 \\
Level 4 & & \\
11 & 15 & 53.3 \\
12 & 14 & 57.1 \\
13 & 8 & 25 \\
14 & 3 & 66.7 \\
15 & 2 & 100 \\
\hline
\end{tabular}

than those who did not have this combination $(24$ of 158 [15.2\%] vs 13 of 200 [6.5\%], respectively; $P=.0073)$.

Before the development of the CP2 score, the time needed to review an entire weekly patient appointment list of approximately 100 patients and provide CMR for selected patients at the Park Meadows Clinic was approximately 6 hours. Utilization of the CP2 score in current practice has reduced this to 1.5 hours weekly.

\section{Discussion}

This is a pilot study evaluating the application of a simple but novel scoring tool. The tool includes patient comorbidities important to a clinical pharmacist using a comprehensive list of ICD-9 codes identified in the patient's medical history or problem list, as well as blood pressure, hemoglobin $\mathrm{A}_{1 \mathrm{c}}$, and glomerular filtration rate. These data can easily be pulled from most EHRs. For this study, information technology professionals developed code to extract data from the EPIC EHR and report the patient list with CP2 scores in an automated fashion. If desiring to use the CP2 score in alternate EHRs, coding would most likely be needed for each individual EHR system. The need to code separately is not anticipated to be a significant barrier to using the $\mathrm{CP} 2$ score given the basic nature of the patient-specific information required.

The CP2 score was reliable in discriminating patients who would benefit from CMR by a clinical pharmacist from those who would not. Our analysis clearly shows that patients with low scores were less likely to have drug therapy problems after CMR by a clinical pharmacist compared with patients with high scores. Identifying drug therapy problems after CMR is most efficient for patients with a high CP2 score $(\geq 11)$. For these patients, the number needed to review (NNR) to identify $\geq 1$ drug therapy problem was 2 . This means that for every 2 patients with a CP2 score of $\geq 11, \mathrm{CMR}$ by the clinical pharmacist resulted in a clinical pharmacy note providing at least 1 medication recommendation. Alternatively stated, $50 \%$ of patients with a

Table 4. Drug Therapy Problems Identified and the Most Common Medication Recommendations ( $\mathrm{n}=$ 223)

\begin{tabular}{|c|c|}
\hline $\begin{array}{l}\text { Drug Therapy Problem } \\
\text { (n) }\end{array}$ & $\begin{array}{c}\text { Most Common } \\
\text { Medication } \\
\text { Recommendation (n) }\end{array}$ \\
\hline \multirow{4}{*}{$\begin{array}{l}\text { Needs additional therapy } \\
\text { (118) }\end{array}$} & Vaccine $(62)$ \\
\hline & $\begin{array}{l}\text { HMG-CoA reductase } \\
\text { inhibitor }(31)\end{array}$ \\
\hline & $\begin{array}{l}\text { Blood pressure-lowering } \\
\text { agent (6) }\end{array}$ \\
\hline & Antidiabetic agent (4) \\
\hline \multirow[t]{2}{*}{ Unnecessary drug (31) } & $\begin{array}{l}\text { ER niacin or ezetimibe } \\
\text { not needed (10) }\end{array}$ \\
\hline & $\begin{array}{l}\text { Triglyceride lowering not } \\
\text { needed (7) }\end{array}$ \\
\hline \multirow[t]{2}{*}{ Wrong dose (24) } & $\begin{array}{l}\text { Blood pressure drug dose } \\
\text { too high/low }(17)\end{array}$ \\
\hline & $\begin{array}{l}\text { Diabetes drug dose too } \\
\text { high/low (3) }\end{array}$ \\
\hline Cost (13) & $\begin{array}{l}\text { Brand switched to } \\
\text { different generic (8) }\end{array}$ \\
\hline $\begin{array}{l}\text { Potentially inappropriate } \\
\text { drug (10) }\end{array}$ & $\begin{array}{l}\text { Unsafe in patient } \geq 65 \\
\text { years old }(10)\end{array}$ \\
\hline Duplicate therapy (7) & $\begin{array}{l}\text { Aspirin/clopidogrel when } \\
\text { not indicated (2) }\end{array}$ \\
\hline Laboratory test needed (6) & Vitamin D (2) \\
\hline Wrong drug (5) & $\begin{array}{l}\text { Five different drugs } \\
\text { identified (5) }\end{array}$ \\
\hline Adverse drug reaction (4) & $\begin{array}{l}\text { Four different reactions } \\
\text { identified (4) }\end{array}$ \\
\hline Drug interaction (4) & Simvastatin interaction (4) \\
\hline Other (1) & Nonadherence (1) \\
\hline
\end{tabular}

ER, extended release. 
CP2 score of $\geq 11$ had a drug therapy problem identified. For patients with CP2 scores of 8 to 10 , the NNR was 3 . Overall, reviewing patient appointments for CP2 scores of $\geq 8$ was highly efficient, resulting in the identification of a high percentage of patients with drug therapy problems.

The NNR for CP2 scores of 3 to 7 was 10 . Individually reviewing this group of patients, which represents less complicated patients, would take significant resources to identify few patient appointments with drug therapy problems. Further exploratory analysis of this group demonstrated that patients with diabetes mellitus or hypertension and either $\geq 6$ items on their medication list or a blood pressure $>140 / 90 \mathrm{mmHg}$ were more likely to receive an intervention than those who did not have this combination. Among patient appointments with CP2 scores of 3 to 7 , the NNR to make a medication recommendation was lower for those with the combination of factors compared with those without $(\mathrm{NNR}=6.6$ vs 15.4 , respectively).

The CP2 score decreased the time it took to provide the clinical pharmacy service by an estimated $75 \%$. This tool may facilitate the inclusion of clinical pharmacists on interprofessional health care teams within the patient-centered medical home by improving efficiency and reducing clinical pharmacist cost. Concluding that a clinical pharmacist could cover multiple smaller primary care clinics (eg, 4 to 5 clinics) and achieve similar results by using the CP2 score is reasonable. Inclusion of clinical pharmacists on interprofessional care teams has been supported by the US surgeon general, who stated that "utilization of pharmacists as an essential part of the health care team to prevent and manage disease in collaboration with other clinicians can improve quality, contain costs, and increase access to care." ${ }^{8}$ Within the patient-centered medical home, clinical pharmacists can provide comprehensive medication review, identify and resolve adverse drug reactions, and recommend costeffective treatments. ${ }^{9}$ Including clinical pharmacists on the health care team has improved patient outcomes in a variety of health care settings and for many disease states. ${ }^{10}$ Despite positive benefits, judicious use of a clinical pharmacist is necessary given the median annual wage of a pharmacist was $\$ 116,670$ in May 2012, or $\$ 56.09$ per hour (not including fringe benefits). ${ }^{11}$

It is important to note that $>25 \%$ of the total recommendations made by the clinical pharmacist were for vaccinations. Of these, $>50 \%$ were for pneumococcal vaccination. Vaccinations are an important health care quality indicator and can also generate revenue for the clinic. The fact that a total of 62 were recommended among all patients reviewed shows that there exists an opportunity to improve care. There were a variety of additional drug therapy recommendations made by the clinical pharmacist; the majority targeted optimal treatment of chronic disease (eg, hypertension, diabetes); the use of unnecessary drugs that can increase cost, increase risk of drug interactions, and increase side effects; and drugs that are inappropriate in the elderly. Targeting and improving these areas of medication use is valuable for patients and the health care system. As noted earlier, previous published work from the Park Meadows Clinic has shown a significant return on investment when evaluating direct savings and the pharmacist cost associated with this service. ${ }^{1}$ The recommendation acceptance rate with this analysis was slightly lower than the previous but still high at approximately $60 \%$. With similar types of recommendations, a similar recommendation acceptance rate, and decreased pharmacist time needed to identify patients who would benefit from CMR, the return on investment would be expected to be even better with use of the CP2 score compared with previous work.

There are limitations to this study. The clinical pharmacist was not blinded to the individual patient CP2 score when providing CMR. This may have introduced bias, causing the clinical pharmacist to identify more drug therapy problems among those with higher scores. There were patients who had $>1$ appointment during the study period and received $C M R \geq 2$ times. These patients may not have had drug therapy problems $\geq 2$ times, may have had drug therapy problems only initially, or may have had drug therapy problems at each visit. Because 1107 patients received CMR over an 11week period, duplicate patient appointments are not likely to have skewed the results. The CP2 scoring tool requires ICD-9 codes documented in the EHR to accurately gather patient information. Incorrect or missing documentation can result in inaccurate scoring. As an example, patients who do not have a reconciled medication list might get a low score because of the inaccurate medication list even though they would benefit from CMR. To show the tool was beneficial, the end point used in this study was the presence or absence of drug 
therapy problems. Whether this end point resulted in a change in health care outcomes is not known, though previous work has demonstrated the value of clinical pharmacists to the health care team. ${ }^{10}$ Finally, the patient-specific factors used to generate the $\mathrm{CP} 2$ score were dictated by the capability of the EHR. For instance, the EPIC EHR within the University of Colorado Hospital system does not use the Cockcroft-Gault equation to determine creatinine clearance, so the estimated glomerular filtration rate was used. ${ }^{12,13}$ The Cockcroft-Gault equation is most commonly used for drug dosing. ${ }^{14}$

A tool that perfectly identifies those who have drug therapy problems compared with those who do not is not likely. The CP2 score was developed to reduce the overall burden of auditing all patient appointments. For this purpose, the tool worked well. Additional research is needed to provide further refinement of the CP2 tool using a panel of clinical pharmacy and medical experts. The refined CP2 score could then be validated by testing it in additional family medicine populations using blinded clinical pharmacists.

\section{Conclusion}

The CP2 score was effective at identifying patients who were more likely to receive a medication recommendation after CMR provided by a clinical pharmacist. Expanding the use of the CP2 score to clinical pharmacists in a large family medicine system could increase efficiency and potentially enhance the delivery of interprofessional care within the patient-centered medical home. Further research refining and validating this tool and evaluating patient-level outcomes when using this system is warranted.

\section{References}

1. Vande Griend JP, Saseen JJ, Bislip D, Moore G, Conry C. An off-site clinical pharmacy service in family medicine: development and 1-year outcomes. Fam Med 2014;46:348-53.
2. Brown SJ. The state of family medicine. Fam Pract Manag 2012;19:7.

3. McGaha AL, Garrett E, Jobe AC, et al. Responses to medical students' frequently asked questions about family medicine. Am Fam Physician 2007;76:99106.

4. Rovers J, Hagel H. Self-assessment tool for screening patients at risk for drug therapy problems. J Am Pharm Assoc (2003) 2012;52:646-52.

5. Doucette WR, Chang EH, Pendergast JF, Wright KB, Chrischilles EA, Farris KB. Development and initial assessment of the medication user self-evaluation (MUSE) tool. Clin Ther 2013;35:344-50.

6. Tracy CS, Bell SH, Nickell LA, Charles J, Upshur RE. The IMPACT clinic: innovative model of interprofessional primary care for elderly patients with complex health care needs. Can Fam Physician 2013; 59:e148-55.

7. Falconer N, Nand S, Liow D, Jackson A, Seddon M. Development of an electronic patient prioritization tool for clinical pharmacist interventions. Am J Health Syst Pharm 2014;71:311-20.

8. Benjamin R. Letter of support. Available from: http:// www.usphs.gov/corpslinks/pharmacy/documents/ 2011SupportLetterFromUSSG.pdf. Accessed July 8, 2014.

9. Smith M, Bates DW, Bodenheimer T, Cleary PD. Why pharmacists belong in the medical home. Health Aff (Millwood) 2010;29:906-13.

10. Chisholm-Burns MA, Kim Lee J, Spivey CA, et al. US pharmacists' effect as team members on patient care: systematic review and meta-analyses. Med Care 2010;48:923-33.

11. Bureau of Labor Statistics, US Department of Labor. Occupational Outlook Handbook, 2014-15 edition. Pharmacists. Available from: http://www.bls.gov/ooh/ healthcare/pharmacists.htm. Accessed May 8, 2014.

12. Cockcroft DW, Gault MH. Prediction of creatinine clearance from serum creatinine. Nephron 1976;16: $31-41$.

13. Levey AS, Coresh J, Greene T, et al. Using standardized serum creatinine values in the modification of diet in renal disease study equation for estimating glomerular filtration rate. Ann Intern Med 2006;145: $247-54$.

14. Dowling TC, Matzke GR, Murphy JE, Burckart GJ. Evaluation of renal drug dosing: prescribing information and clinical pharmacist approaches. Pharmacotherapy 2010;30:776-86. 